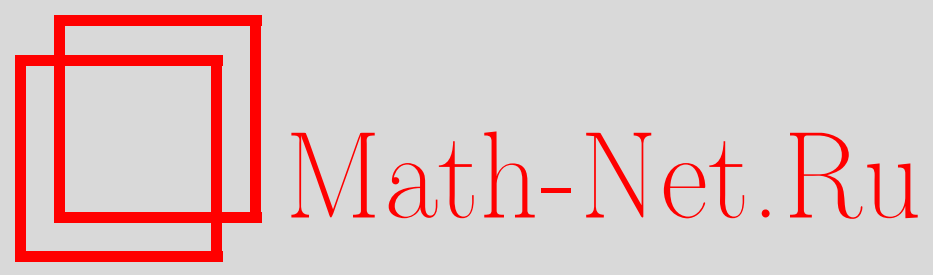

И. В. Аржанцев, О стабильности диагональных действий, Матем. заметки, 2002, том 71, выпуск 6, 803-806

DOI: https://doi.org/10.4213/mzm385

Использование Общероссийского математического портала Math-Net.Ru подразумевает, что вы прочитали и согласны с пользовательским соглашением http://www.mathnet.ru/rus/agreement

Параметры загрузки:

IP: 35.174 .16 .151

26 апреля 2023 г., 13:53:55

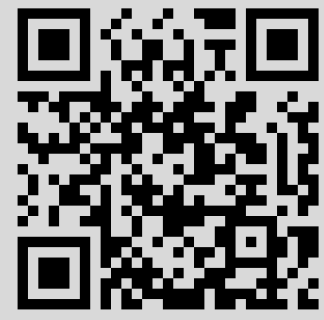




\title{
О СТАБИЛЬНОСТИ ДИАГОНАЛЬНЫХ ДЕЙСТВИЙ
}

\section{И. В. Аржанцев}

\begin{abstract}
В заметке доказано, что для произвольного действия полупростой группы $G$ на афффинном многообразии $X$ найдется натуральное число $n$ такое, что диагональное действие $G: X \times X \times \cdots \times X$ ( $m$ копий $)$ стабильно для любого $m \geqslant n$.

Библиография: 3 названия.
\end{abstract}

Пусть $G$ - полупростая алгебраическая группа над алгебраически замкнутьп полем $k$ нулевой характеристики. Рассмотрим регулярное действие группы $G$ на неприводимом аффинном алгебраическом многообразии $X$, также определенном над полем $k$. Действие $G: X$ называется стабильны.м, если типичная $G$-орбита на $X$ замкнута, или, более точно, если в $X$ найдется непустое открытое подмножество $X_{0}$, для каждой точки которого ее $G$-орбита замкнута в $X$. Свойство стабильности играет важную роль в современной теории инвариантов, поскольку для стабильных действий и только для них типичньй слой морфизма факторизации состоит из одной орбиты.

Известно несколько критериев и достаточных условий стабильности действия, см. например $[1$, п. 7.5$]$. Ниже мы рассмотрим один из результатов в этом направлении. Будем обозначать через $V_{\lambda}$ неприводимый $G$-модуль со старшим весом $\lambda$. Старший вес сопряженного $G$-модуля $V_{\lambda}^{*}$ обозначим через $\lambda^{*}$. Пусть $\theta$ - инволюция Вейля на группе $G$, т.е. инволютивньй автоморфизм, действующий как инверсия на некотором максимальном торе. Известно, что для любого представления $R$ группы $G \theta$-подкрученное представление $R \circ \theta$ изоморфно сопряженному представлению $R^{*}$. Пусть $G$ действует на неприводимом аффинном многообразии $X$. Обозначим через $X^{*}$ то же многообразие, но с $\theta$-подкрученным действием. Тогда диагональное действие $G: X \times X^{*}$ стабильно $[2],[3]$.

Если каждое представление групшы $G$ самосопряжено, то диагональное действие $G: X \times X$ стабильно для любого аффинного $G$-многообразия $X$. Для произвольной полупростой группы это не всегда так. Например, рассмотрим тавтологическое линейное действие $S L(n): k^{n}$. При $n>2$ диагональное действие $S L(n): k^{n} \times k^{n}$ не является стабильным. Однако диагональное действие $S L(n): k^{n} \times k^{n} \times \cdots \times k^{n}$ ( $m$ копий) стабильно при $m \geqslant n$. Цель настоящей заметки - показать, что это явление имеет место для произвольного действия.

Всюду далее будем обозначать $X^{m}=X \times X \times \cdots \times X(m$ копий $)$ и действие $G: X^{m}$ считать диагональным.

Работа выполнена при финансовой поддержке Российского фонда фундаментальных исследований, грант № 01-01-00756, и фонда CRDF, грант № RM1-2088. 
ТЕорема 1. Для произвольного действия $G: X$ полупростой группы $G$ на неприводимом аффинном многообразии $X$ найдется натуральное число $п$ такое, что действие $G: X^{m}$ стабильно для любого $m \geqslant n$.

ДокАЗАТЕЛЬСтво. Этап 1. Без ограничения общности можно предполагать, что группа $G$ связна и действие $G: X$ эффективно.

Будем говорить, что для действия $G$ на $X$ существует стабилизатор общего положения (с.о.п.) $G_{*}$, если $G_{*}$ - такая подгруппа в $G$, что для некоторого открытого подмножества $X_{0} \subset X$ стабилизатор любой точки из $X_{0}$ сопряжен $G_{*}$.

Лемма 1. Для произвольного әффективного действия алгебраической группы $G$ на алгебраическом многообразии $X$ существует натуральное число $k$ такое, что для любого $l>k$ для действия $G: X^{l}$ c.о.n. существует и является единичной подгруппой.

ДокАЗАТЕльство. Покажем, что для некоторого $k$ в $X^{k}$ найдется точка, стабилизатор которой есть единичная подгруппа. Будем рассуждать от противного. Пусть стабилизатор точки $\left(x_{1}, \ldots, x_{s}\right) \in X^{s}$ есть подгруппа $H_{s}$. В силу эффективности действия если $H_{s} \neq\{e\}$, то найдется точка $x_{s+1} \in X$, стабилизатор которой не содержит $H_{s}$. Тогда стабилизатор $H_{s+1}$ точки $\left(x_{1}, \ldots, x_{s+1}\right) \in X^{s+1}$ есть собственная подгруппа в $H_{s}$. Получена бесконечная строго убьвающая цепочка подгрупп алгебраической групшы, что приводит к противоречию.

Пусть $x_{0} \in X^{k}-$ точка с единичньм стабилизатором. Тогда с.о.п. для действия $G: X^{k}$ конечен, см. [1, п. 7.1]. Пусть $X_{0}$ - открытое подмножество в $X^{k}$, стабилизаторы точек которого конечны. Фиксируем точку $x_{1} \in X_{0}$. В силу эффективности действия множество точек $x_{2}$ в $X^{q}$, стабилизаторы которых пересекаются со стабилизатором $G_{x_{1}}$ только по единице, открыто и плотно в $X^{q}$ при $q \geqslant 1$. Поэтому множество точек с единичным стабилизатором плотно в $X^{k+q}$, откуда и следует, что с.о.п. для действия $G: X^{l}, l>k$, есть единичная подгруппа.

Этап 2. Пусть $\Xi_{+}(G)$ - полугрупша доминантных весов групшы $G$. Для конечнопорожденной подполугруппы $M \subset \Xi_{+}(G)$ будем обозначать через $C(M)$ минимальньй выпукльй рациональньй конус, содержащий $M$.

Действие $G: X$ определяет линейное представление группы $G$ в алгебре регулярных функций $k[X]$. Рассмотрим изотипное разложение

$$
k[X]=\bigoplus_{\lambda \in \Xi_{+}(G)} k[X]_{\lambda} .
$$

Для неприводимого многообразия $X$ множество весов $\Xi(G, X)=\left\{\lambda \in \Xi_{+}(G) \mid k[X]_{\lambda}\right.$ $\neq 0\}$ образует подполугруппу в $\Xi_{+}(G)$. В частности, такая полугруппа $\Xi(G, G / H)$ связана с каждым однородным пространством $G / H$.

Теорема 2 (Э.Б. Винберг [3, теорема 10]). Пусть действие $G: X$ может быть продолжено до действия большей связной редуктивной группы $F \supset G$. Предположим, чтоо

$$
\Xi(F, X)-\Xi(F, F / G)=\left\{\lambda_{1}-\lambda_{2} \mid \lambda_{1} \in \Xi(F, X), \lambda_{2} \in \Xi(F, F / G)\right\}
$$


является группой. Тогда действие $G: X$ стабильно.

Заметим, что для двух полугрупп $\Omega_{1}, \Omega_{2} \subset \mathbb{Z}^{n} \subset \mathbb{R}^{n}$ условие " $\Omega_{1}-\Omega_{2}$ является группой" эквивалентно условию "конусы $C\left(\Omega_{1}\right)$ и $C\left(\Omega_{2}\right)$ имеют общую внутреннюю точку".

Мы применим теорему 2 для действия $G: X^{n}$, полагая $F=G^{n}$ с покомпонентным действием $G^{n}: X^{n}$.

Согласно лемме 1 , заменяя $X$ на $X^{k}$, можно считать, что в $X$ содержится точка $x_{0}$ с единичным стабилизатором. Пусть $Y=\overline{G x_{0}}$. Многообразие $Y$ эквивариантно бирационально изоморфно групе $G$. Поэтому полугруппа $\Xi(G, Y)$ содержит внутреннюю точку доминантной камеры Вейля $C\left(\Xi_{+}(G)\right)$. Вложение $Y \subset X$ определяет вложение $\Xi(G, Y) \subset \Xi(G, X)$. Пусть $\lambda_{0} \in \Xi(G, X)$ - внутренняя точка камеры Вейля, являющаяся также внутренней точкой конуса $C(\Xi(G, X))$. Обозначим точку $(\lambda, \ldots, \lambda) \in X^{n}$ символом $\lambda^{n}$. При любом $n$ точка $\lambda_{0}^{n}$ является внутренней для конуса $C\left(\Xi\left(G^{n}, X^{n}\right)\right)=$ $C(\Xi(G, X))^{n}$. Нужно подобрать $n$ так, чтобы $\lambda_{0}^{n}$ была внутренней точкой конуса $C\left(\Xi\left(G^{n}, G^{n} / G\right)\right)$.

По двойственности Фробениуса $[1$, п. 3.7$]$ имеем

$$
\begin{aligned}
\Xi\left(G^{n}, G^{n} / G\right)= & \left\{\left(\lambda_{1}, \ldots, \lambda_{n}\right) \mid G \text {-модуль } V_{\lambda_{1}} \otimes \cdots \otimes V_{\lambda_{n}}\right. \text { содержит } \\
& \text { ненулевой } G \text {-инвариантньй вектор }\} .
\end{aligned}
$$

ЛЕмма 2. Для любого $\lambda \in \Xi_{+}(G)$ найдется $p$, для которого $\lambda^{p} \in \Xi\left(G^{p}, G^{p} / G\right)$.

ДоКАЗАТЕЛЬСТво. Поскольку $G \subset S L\left(V_{\lambda}\right)$, достаточно указать ненулевой $S L(V)$ инвариантньй вектор в $V \otimes \cdots \otimes V$ для некоторого числа экземпляров $V$. Таким вектором является тензор det.

Пусть $\omega_{1}, \ldots, \omega_{r}-$ фундаментальные веса групшы $G$, и пусть число $n$ таково, что $\lambda_{0}^{n}, \omega_{1}^{n}, \ldots, \omega_{r}^{n} \in \Xi\left(G^{n}, G^{n} / G\right)$. Для того чтобы проверить, что точка $\lambda_{0}^{n} \in C\left(\Xi\left(G^{n}\right.\right.$, $\left.\left.G^{n} / G\right)\right)$ является внутренней, нужно показать, что для любой линейной функции $l$ в $\mathbb{R}^{r n}$ такой, что $l\left(\lambda_{0}^{n}\right)=0$ и $\left.l\right|_{C\left(\Xi\left(G^{n}, G^{n} / G\right)\right)} \geqslant 0$, имеем $\left.l\right|_{C\left(\Xi\left(G^{n}, G^{n} / G\right)\right)} \equiv 0$. Из равенства $\lambda_{0}^{n}=k_{1} \omega_{1}^{n}+\cdots+k_{r} \omega_{r}^{n}, k_{i}>0$, следует $l\left(\lambda_{0}^{n}\right)=k_{1} l\left(\omega_{1}^{n}\right)+\cdots+k_{r} l\left(\omega_{r}^{n}\right)$ и, значит, $l\left(\omega_{i}^{n}\right)=0$ и $l\left(\lambda^{n}\right)=0$ для всех $\lambda \in \Xi\left(G^{n}, G^{n} / G\right)$. Если $\left(\lambda_{1}, \ldots, \lambda_{n}\right) \in \Xi\left(G^{n}, G^{n} / G\right)$, то и $\left(\lambda_{\tau(1)}, \ldots, \lambda_{\tau(n)}\right) \in \Xi\left(G^{n}, G^{n} / G\right)$ для всякой подстановки $\tau \in S_{n}$. Применяя к набору $\left(\lambda_{1}, \ldots, \lambda_{n}\right)$ циклические перестановки и суммируя, получаем $\left(\lambda_{1}+\cdots+\lambda_{n}\right)^{n} \in \Xi\left(G^{n}\right.$, $\left.G^{n} / G\right)$. Но из условия $l\left(\left(\lambda_{1}+\cdots+\lambda_{n}\right)^{n}\right)=0$ следует $l\left(\lambda_{1}, \ldots, \lambda_{n}\right)=0$, что и требовалось доказать.

Этап 3. На предыдущих этапах мы нашли номер $n$, для которого действие $G: X^{n}$ имеет единичный с.о.п. и стабильно. Стабильность действия $G: X^{m}$ при $m>n$ вытекает из следующей леммы.

Лемма 3. Пусть действие $G: Z_{1}$ редуктивной группы $G$ на аффинном многообразии $Z_{1}$ имеет конечный с.о.п. и стабильно. Тогда для произвольного действия $G: Z_{2}$ на аффинном многообразии $Z_{2}$ диагональное действие $G:\left(Z_{1} \times Z_{2}\right)$ стабильно.

ДокАЗАТЕЛЬСТВо. Пусть $z_{1}$ - точка на $Z_{1}$ с конечным стабилизатором, орбита которой замкнута. Покажем, что для любой точки $z_{2} \in Z_{2}$ орбита точки $\left(z_{1}, z_{2}\right)$ замкнута 
в $Z_{1} \times Z_{2}$. В самом деле, в замыкании орбиты $G\left(z_{1}, z_{2}\right)$ лежит (единственная) замкнутая $G$-орбита $\mathscr{O}[1$, п. 4.4]. Теорема Гильберта-Мамфорда-Биркса $[1$, п. 6.8] утверждает, что найдется однопараметрическая подгруппа $\mu: k^{*} \rightarrow G$, для которой предельная точка $\lim _{t \rightarrow 0} \mu(t)\left(z_{1}, z_{2}\right)$ лежит в $\mathscr{O}$. В этой ситуации точка $z_{0}=\lim _{t \rightarrow 0} \mu(t) z_{1}$ лежит в замькании $G z_{1}$, а значит, и в самой $G z_{1}$. С другой стороны, стабилизатор точки $z_{0}$ содержит подгруппу $\mu(t)$. В силу конечности стабилизатора $G_{z_{0}}$ подгруппа $\mu(t)$ совпадает с единичной подгруппой и $G\left(z_{1}, z_{2}\right)=\mathscr{O}$.

Теорема 1 полностью доказана.

СлЕДСТВИЕ 1. Для произвольного действия $G: X$ полупростой әруппы $G$ на неприводимом аффинном многообразии $X$ найдется натуральное число $n$ такое, что полугруппа $\Xi\left(G, X^{m}\right)$ совпадает $c \Xi_{+}(G)$ для всех $m \geqslant n$.

ДокАЗАТЕЛЬСТво. Из теоремы 1 и леммы 1 вытекает, чтопри $m \geqslant n$ в $X^{m}$ найдется точка $x_{0}$, стабилизатор которой единичен, а орбита замкнута. Имеем $\overline{G x_{0}} \cong G$, откуда $\Xi\left(G, \overline{G x_{0}}\right)=\Xi_{+}(G)$. Вложение $\Xi\left(G, \overline{G x_{0}}\right) \subseteq \Xi\left(G, X^{m}\right)$ завершает доказательство.

В заключение отметим, что теорема 1 не может быть перенесена на действия редуктивных групш. Простейший пример доставляет действие одномерного тора гомотетиями на прямой.

Автор благодарен профессору Э. Б. Винбергу за ценные замечания.

\section{СПИСОК ЦИТИРОВАННОЙ ЛИТЕРАТУРЫ}

[1] Винберг Э. Б., Попов В. Л. Теория инвариантов // Итоги науки и техники. Совр. проблемы матем. Фундамент. направления. Т. 55. М.: ВИНИТИ, 1989. С. 137-314.

[2] Panyushev D. I. A restriction theorem and the Poincaré series for $U$-invariants // Math. Ann. 1995. V. 301. P. 655-675.

[3] Vinberg E. B. On stability of actions of reductive algebraic groups // Lie Algebras, Rings and Related Topics / ed. Fong Yuen, A. A. Mikhalev, E. Zelmanov. Hong-Kong: Springer-Verlag, 2000. P. 188-202. 